Revista Brasileira de Agricultura Irrigada v.11, nº.7, p. 1998 - 2009, 2017

ISSN 1982-7679 (On-line)

Fortaleza, CE, INOVAGRI - http://www.inovagri.org.br

DOI: $10.7127 /$ rbai.v11n700667

Protocolo 667.17 - 24/04/2017 Aprovado em 26/07/2017

\title{
ESTABILIDADE TEMPORAL E VARIABILIDADE ESPACIAL DA UMIDADE DE UM ARGISSOLO CULTIVADO COM PALMA FORRAGEIRA
}

Ailton Alves de Carvalho ${ }^{1}$, Thieres George Freire da Silva ${ }^{2}$, Maria Gabriela de Queiroz ${ }^{3}$, George do Nascimento Araújo Júnior ${ }^{4}$, Alexandre Maniçoba da Rosa Ferraz Jardim ${ }^{4}$, Carlos André Alves de Souza ${ }^{4}$

\section{RESUMO}

A umidade do solo é uma variável de grande importância para o manejo de água em cultivos agrícolas. O uso recente de irrigação em áreas de palma forrageira induz a necessidade de mensuração da umidade do solo para auxílio da tomada de decisão de quando e quanto irrigar. Logo, a estabilidade temporal e a variabilidade espacial da umidade do solo em um argissolo vermelho amarelo cultivado com palma forrageira foram avaliadas neste estudo. A pesquisa foi conduzida em Serra Talhada, PE, em um cultivo de palma forrageira com dimensões de $76 \mathrm{x}$ $32 \mathrm{~m}$ e declividade diagonal de $0,6 \%$, onde medidas da umidade no perfil do solo foram efetuadas em 60 posições (pontos amostrais equidistantes em $7 \mathrm{~m}$ ), a cada 0,10 m, em seis camadas, até a profundidade de 0,60 m e em intervalos de três dias durante 41 dias. Estatística descritiva, diferença relativa da posição, matriz de correlação de Spearman e geoestatística foram aplicadas. A umidade ao longo do perfil do solo demonstrou dispersão mediana (1020\%), mesmo depois da realização de irrigação ou ocorrência de eventos de chuva. A técnica da diferença relativa da posição mostrou que a dispersão espacial da umidade do solo dependeu da camada. A matriz de correlação de Spearman detectou estabilidade temporal da umidade em todas as camadas analisadas, porém mais intensa nas maiores profundidades. A geoestatística revelou dependência entre a umidade do solo cultivado com palma e a cota da posição de medição, e estreita relação aos pontos com maior estabilidade temporal.

Palavras-chave: geoestatística, irrigação, Opuntia stricta (Haw.) Haw., Semiárido

\section{TEMPORAL STABILITY AND SPATIAL VARIABILITY OF SOIL MOISTURE IN A ARGISSOL CULTIVATED WITH FORAGE CACTUS}

\footnotetext{
${ }^{1}$ Doutorando em Engenharia Agrícola, Universidade Federal Rural de Pernambuco, Recife-PE, e-mail: ailtonalvesst@hotmail.com

${ }^{2}$ Prof. Dr. em Meteorologia Agrícola, Universidade Federal Rural de Pernambuco, Unidade Acadêmica de Serra Talhada, Serra Talhada-PE, e-mail: thieres_freire@yahoo.com.br

${ }^{3}$ Doutoranda em Meteorologia Agrícola, Universidade Federal de Viçosa, e-mail: mg.gabi@hotmail.com

${ }^{4}$ Mestrando do Programa de Pós-graduação em Produção Vegetal, Universidade Federal Rural de Pernambuco, Unidade Acadêmica de Serra Talhada, Serra Talhada-PE, e-mail: georgejunior_91@hotmail.com, alexandremrjj@gmail.com, carlosandre08_@msn.com
} 


\begin{abstract}
Soil moisture is a variable of great importance for the management of water in agricultural crops. The recent use of irrigation in forage cactus areas induces the need to measure soil moisture to aid decision making when and how much to irrigate. Therefore, temporal stability and spatial variability of soil moisture in a yellow red argisol cultivated with forage cactus were evaluated in this study. The experiment was conducted in municipality of Serra Talhada, State of Pernambuco, in a forage cactus area with dimensions of $76 \times 32 \mathrm{~m}$ and diagonal slope of $0.6 \%$, where soil moisture measurements were carried out in 60 positions (sampling points equidistant in $7 \mathrm{~m}$ ), every $0.10 \mathrm{~m}$, in six layers, until depth of $0.60 \mathrm{~m}$ and at intervals of three days for 41 days. Descriptive statistics, position relative difference, Spearman correlation matrix and geostatistics were applied. The moisture along soil profile showed a medium dispersion (10-20\%) even after irrigation or rain events. The technique of the position relative difference showed that spatial dispersion of soil moisture depended on the layer. The Spearman correlation matrix detected temporal stability of the moisture in all analyzed layers, but more intense in the greater depths. Geostatistics revealed dependence between moisture of the soil cultivated with forage cactus and measurement position, and a close relation to the points with greater temporal stability.
\end{abstract}

Keywords: geostatistics, irrigation, Opuntia stricta (Haw.) Haw., Semiarid

\section{INTRODUÇÃO}

No Brasil, a palma visa à subsistência dos rebanhos, sendo considerada uma das principais forrageiras da região Nordeste, sobretudo nos estados de Alagoas e Pernambuco.

Apesar do seu ótimo desempenho em regiões com baixo nível pluviométrico, o uso de irrigação torna-se uma opção para incrementar expressivamente o rendimento anual da cultura (HERNÁNDEZ et al., 2004; SILVA et al., 2015a; QUEIROZ et al., 2016).

O aumento das áreas cultivadas com palma irrigada induz a necessidade de mensuração da umidade do solo $(\theta)$, como uma estratégia de manejo de água no sistema de produção. A $\theta$ é uma variável responsável pelo controle da troca de água e de energia entre a superfície terrestre e a atmosfera (JIA et al., 2017). Do ponto de vista hidrológico e ecológico, ela condiciona os processos de escoamento superficial, infiltração, armazenamento, drenagem e transpiração das plantas (ZHANG; SHAO, 2013; CHEN et al., 2014; HE et al., 2017). Além disso, auxilia no manejo de irrigação, sobretudo em áreas sob alta demanda evaporativa, onde é preciso utilizar-se de técnicas de conservação do solo e da água (SOUZA et al., 2011; ÁVILA et al., 2011).

Para a conservação da umidade do solo de forma adequada, a sua mensuração deve ser realizada nos pontos que melhor representam a distribuição no tempo e no espaço (HU et al., 2010). Diversos trabalhos foram conduzidos sobre a variação espaço-temporal da umidade do solo em áreas agrícolas (MELO FILHO; LIBARDI, 2005; HU et al., 2010; ÁVILA et al., 2011; ZHANG; SHAO, 2013; RÖTZER; MONTZKA; VEREECKEN, 2015). Porém, não existem estudos que demonstrem essa análise em áreas de cultivo de palma forrageira.

O movimento da água no solo é muito heterogêneo e depende da ação de diversos fatores relacionados ao clima, solo, topografia e vegetação, resultando em uma alta variabilidade espacial e temporal (ÁVILA; MELLO; SILVA, 2010; ZUCCO et al., 2014). $O$ conhecimento da variabilidade da $\theta$ é importante para o manejo racional da água e a maximização da produtividade (MOREIRA et al., 2014; PENG; MU; SUN, 2017).

Para a análise espaço-temporal da umidade do solo é necessário a coleta de dados 
em diferentes pontos da área, o que, em muitos casos, a quantidade destes pode ser elevada, aumentando os custos de produção. Logo, Vachaud et al. (1985) sugerem duas técnicas para a redução do número de amostras de umidade do solo, sem prejudicar a distribuição dos dados: a primeira, denomina-se de diferença relativa $\left(\delta_{\mathrm{ij}}\right)$, a qual faz uma análise dos desvios entre os valores observados de forma individual no espaço e a média entre eles; quando a diferença relativa apresenta pequena variação temporal entre as posições, havendo indicação de estabilidade temporal (MOREIRA et al., 2014; ZUCCO et al., 2014). Na segunda técnica é aplicado o teste não paramétrico de Spearman, que indica o grau de concordância da variabilidade espacial em diferentes tempos; quanto mais próximo de 1 , o coeficiente de correlação indica estabilidade existente entre os tempos (MELO FILHO; LIBARDI, 2005; LEMOS FILHO, 2010). As duas técnicas citadas são utilizadas na análise da estabilidade temporal da umidade. Por outro lado, a variação espacial pode ser analisada por meio da geoestatística, que está associada a uma classe de técnicas que permite inferir os valores de uma ou mais variáveis (CASTRO et al., 2010), mapeando fenômenos contínuos, por meio da interpolação dos pontos amostrados no espaço e no tempo.

Objetivou-se avaliar a estabilidade temporal e a variabilidade espacial da umidade do solo em um cultivo de palma forrageira irrigada no Semiárido brasileiro.

\section{MATERIAL E MÉTODOS}

O experimento foi conduzido no município de Serra Talhada, PE (latitude: $7^{\circ}$ 56' 20', S; longitude: $38^{\circ} 17^{\prime} 31^{\prime \prime} \mathrm{O}$; e, altitude: $498 \mathrm{~m}$ de altitude). O clima da região é semiárido do tipo BSwh, segundo a classificação de Köppen. A precipitação média anual é de $642 \mathrm{~mm}$, concentrada entre os meses de janeiro a abril, e temperatura média de 24,8 ${ }^{\circ} \mathrm{C}$ (SILVA et al., 2015b). O solo na área de estudo foi caracterizado como Argissolo Vermelho Amarelo (EMBRAPA, 2013), com as propriedades físicas do solo apresentadas na Tabela 1.

Tabela 1. Propriedades físicas de um argissolo vermelho amarelo cultivado com palma forrageira

\begin{tabular}{ccccccc}
\hline $\begin{array}{c}\text { Camada } \\
\mathrm{m}\end{array}$ & $\begin{array}{c}\rho_{\mathrm{s}} \\
\mathrm{kg} \mathrm{dm}^{-3}\end{array}$ & $\begin{array}{c}\rho_{\mathrm{p}} \\
\mathrm{kg} \mathrm{dm}^{-3}\end{array}$ & $\begin{array}{c}\varphi_{\mathrm{t}} \\
\%\end{array}$ & $\begin{array}{c}\text { Areia } \\
\mathrm{g} \mathrm{kg}^{-1}\end{array}$ & $\begin{array}{c}\text { Silte } \\
\mathrm{g} \mathrm{kg}^{-1}\end{array}$ & $\begin{array}{c}\text { Argila } \\
\mathrm{g} \mathrm{kg}^{-1}\end{array}$ \\
\hline $0,00-0,10$ & 1,52 & 2,55 & 40,48 & 719,80 & 263,40 & 16,80 \\
$0,10-0,20$ & 1,62 & 2,57 & 37,11 & 719,20 & 247,30 & 33,60 \\
$0,20-0,30$ & 1,59 & 2,60 & 38,92 & 710,80 & 248,30 & 40,80 \\
$0,30-0,40$ & 1,54 & 2,55 & 39,48 & 645,20 & 279,10 & 75,80 \\
$0,40-0,50$ & 1,49 & 2,59 & 42,58 & 607,00 & 297,90 & 95,10 \\
$0,50-0,60$ & 1,42 & 2,63 & 46,00 & 575,00 & 304,60 & 120,40 \\
\hline
\end{tabular}

$\rho_{\mathrm{s}}$ - Densidade do solo $\left(\mathrm{kg} \mathrm{dm}^{-3}\right) ; \rho_{\mathrm{p}}$ - Densidade das partículas $\left(\mathrm{kg} \mathrm{dm}^{-3}\right) ; \varphi_{\mathrm{t}}$ - Porosidade total do solo (\%).

A área com dimensões de 76 x 32 m foi implantada com palma forrageira (Opuntia stricta (Haw.) Haw.), clone Orelha de Elefante Mexicana/IPA-200016, em março de 2011, com plantas espaçadas em 1,6 x 0,4 m, conduzidas sob sistema de plantio exclusivo. A primeira colheita foi realizada em 15 de maio de 2012, procedendo-se um corte de uniformização, quando se manteve apenas os cladódios basais.

Entre os dias 16 e 31 de maio de 2012 foram instalados 60 tubos de acesso de PVC (pontos amostrais), regularmente distribuídos em distâncias de $7 \mathrm{~m}$, com o intuito de monitorar a $\theta$. Cada tubo foi disposto a $0,10 \mathrm{~m}$ das fileiras de cultivo. A instalação foi feita com o auxílio de um tripé, acessórios e ferramentas (haste de silicone, martelo de borracha, trado do tipo holandês, espátula e anéis de latão biselados), seguindo recomendações do fabricante (SENTEK, 2000), de forma a garantir maior verticalidade de inserção do tubo no solo e evitar formação de bolsões de ar e variações na densidade do solo. Logo após as suas instalações, nas extremidades de cada tubo foram inseridos 
tampões para garantir que os mesmos ficassem hermeticamente fechados. A disposição dos tubos de acesso na área de estudo é demostrada na Figura 1.

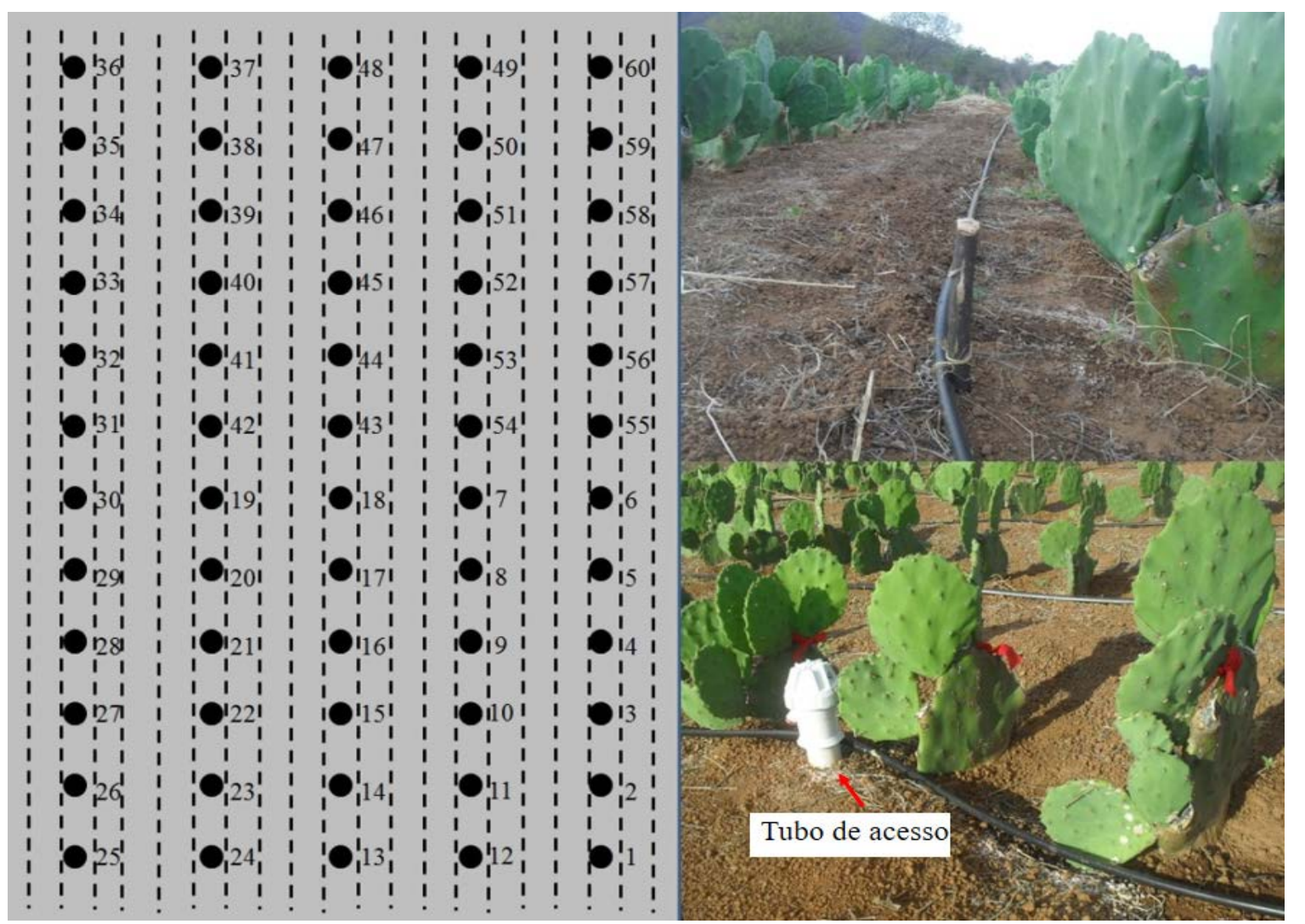

Figura 1. Disposição dos tubos de acesso instalados em um Argissolo Vermelho Amarelo cultivado com palma forrageira. Os pontos de 1 a 60 representam a posição dos tubos de acesso na área de cultivo

A partir do dia 01 de junho de 2012 foi iniciado o período experimental do presente estudo, com término em 11 de julho de 2012, quando totalizaram se 41 dias de avaliações de $\theta$. Ao longo do tempo, foram realizadas leituras de frequência relativa (FR) por meio de uma sonda capacitiva modelo Diviner $2000^{\circledR}$ (Sentek Pty Ltd., Austrália). As leituras de frequência relativa foram medidas a cada 0,10 $\mathrm{m}$ até uma profundidade de $0,60 \mathrm{~m}$, nas segundas-feiras, quartas-feiras e sextas-feiras, sempre antes da irrigação. A sonda foi calibrada nas condições de solo local, de acordo com Araújo Primo et al. (2015), sendo a determinação da $\theta\left(\mathrm{m}^{3} \mathrm{~m}^{-3}\right)$ obtida a partir da equação:

$$
\theta=0,4453 \mathrm{FR}^{2,9403}
$$

em que,

FR é a frequência relativa ( $\mathrm{MHz})$
As práticas de irrigação tiveram início no dia 18 de junho de 2012. Para isso foi utilizado um sistema de irrigação por gotejamento, com emissores espaçados em $0,4 \mathrm{~m}$, vazão nominal de $1,5 \mathrm{~L} \mathrm{~h}^{-1}$ e coeficiente de uniformização de $92 \%$. As lâminas de irrigação foram calculadas com base na evapotranspiração de referência (ETo) e coeficiente de cultura $(\mathrm{kc})$, conforme sugerido por Queiroz et al. (2016). A ETo foi estimada por meio do método de Penman Monteith parametrizado no boletim 56 da FAO (ALLEN et al., 1998) e de dados das variáveis meteorológicas coletados por uma plataforma de coleta de dados (PCD), localizada a $700 \mathrm{~m}$ da área experimental, pertencente ao Instituto Nacional de Meteorologia (INMET).

Os dados de $\theta$ foram tratados de acordo com estatística descritiva e submetidos ao teste de Kolmogorov-Smirnov para análise da normalidade de seus resíduos. O grau de variabilidade dos valores dos coeficientes de variação (CV) foi analisado conforme a 
classificação proposta por Warrick e Nielsen (1980): baixa variabilidade (CV < 12\%), média variabilidade $(12 \leq \mathrm{CV} \leq 62 \%)$ e alta variabilidade $(\mathrm{CV}>62 \%)$.

A estabilidade temporal da $\theta$ foi avaliada por meio da técnica proposta por Vachaud et al. (1985) e pelo coeficiente de correlação de Spearman. O primeiro considera a $\theta$ de cada tubo de acesso (posição i) e tempo (j) em relação à média de $\theta$ de todos os pontos de medição, por camada $(\mathrm{k})$ :

$$
\delta_{\mathrm{ijk}}(\theta)=\frac{\theta_{\mathrm{ijk}}-\overline{\theta_{\mathrm{ijk}}}}{\bar{\theta}_{\mathrm{ijk}}} 100
$$

em que,

$\delta_{\mathrm{ijk}}(\theta)$ é a diferença relativa na posição i no tempo $\mathrm{j}$, na camada $\mathrm{k}$

$\theta_{\mathrm{ijk}}$ é o valor da $\theta$ na posição i no tempo j, na camada $\mathrm{k}$

$\overline{\theta_{\mathrm{ij}}}$ é a média da $\theta$, em todas as posições i no tempo $\mathrm{j}$ da camada $\mathrm{k}$, determinada pela equação:

$$
\overline{\theta_{\mathrm{ijk}}}=\frac{1}{\mathrm{~N}} \sum_{\mathrm{i}=1}^{\mathrm{N}} \theta_{\mathrm{ijk}}
$$

em que,

$\theta_{\mathrm{ijk}}$ é a $\theta$ da posição i no tempo j da camada $\mathrm{k}$ $\mathrm{N}$ é o número de observações

O coeficiente de correlação de Spearman $\left(\mathrm{r}_{\mathrm{s}}\right)$ foi empregado para avaliar o grau de correlação da variabilidade $\theta$ em diferentes tempos de amostragem, de cada camada k:

em que,

$$
r_{s}=1-\frac{6 \sum_{\mathrm{i}=1}^{\mathrm{n}}\left(\theta_{\mathrm{ijk}}-\theta_{\mathrm{ij} \mathrm{j}^{\prime} \mathrm{k}}\right)^{2}}{\mathrm{~N}\left(\mathrm{~N}^{2}-1\right)}
$$

Tabela 2. Nível de significância do Teste de Normalidade de Kolmogorov-Smirnov (Lilliefors) para os dados de umidade do solo monitorados em seis camadas do solo ao longo de 41 dias após o corte da palma forrageira.

\begin{tabular}{ccccccc} 
DAC & $0,00-0,10 \mathrm{~m}$ & $0,10-0,20 \mathrm{~m}$ & $0,20-0,30 \mathrm{~m}$ & $0,30-0,40 \mathrm{~m}$ & $0,40-0,50 \mathrm{~m}$ & $0,50-0,60 \mathrm{~m}$ \\
\hline 1 & 0,025 & 0,039 & 0,001 & $0,200^{*}$ & $0,200^{*}$ & $0,200^{*}$ \\
4 & 0,014 & 0,011 & 0,000 & $0,200^{*}$ & $0,200^{*}$ & $0,200^{*}$ \\
6 & $0,200^{*}$ & 0,047 & 0,000 & $0,200^{*}$ & $0,200^{*}$ & $0,200^{*}$ \\
8 & 0,027 & 0,069 & 0,003 & $0,200^{*}$ & $0,200^{*}$ & $0,200^{*}$ \\
11 & 0,059 & 0,031 & 0,000 & $0,200^{*}$ & $0,200^{*}$ & $0,200^{*}$ \\
13 & $0,200^{*}$ & 0,045 & 0,001 & $0,200^{*}$ & $0,200^{*}$ & $0,200^{*}$
\end{tabular}


Carvalho et al.

\begin{tabular}{lcccccc}
15 & $0,200^{*}$ & 0,085 & 0,000 & $0,200^{*}$ & $0,200^{*}$ & $0,200^{*}$ \\
18 & 0,004 & 0,078 & 0,001 & $0,200^{*}$ & $0,200^{*}$ & $0,200^{*}$ \\
20 & 0,023 & 0,021 & 0,000 & $0,200^{*}$ & $0,200^{*}$ & 0,088 \\
22 & 0,037 & 0,025 & 0,000 & $0,200^{*}$ & $0,200^{*}$ & 0,086 \\
25 & 0,055 & 0,014 & 0,000 & $0,200^{*}$ & $0,200^{*}$ & $0,200^{*}$ \\
27 & $0,200^{*}$ & 0,029 & 0,001 & $0,200^{*}$ & $0,200^{*}$ & $0,200^{*}$ \\
29 & 0,000 & 0,191 & 0,001 & $0,200^{*}$ & $0,200^{*}$ & $0,200^{*}$ \\
32 & 0,000 & 0,088 & 0,000 & $0,200^{*}$ & $0,200^{*}$ & $0,200^{*}$ \\
34 & 0,000 & 0,073 & 0,000 & $0,200^{*}$ & $0,200^{*}$ & $0,200^{*}$ \\
36 & 0,000 & 0,031 & 0,000 & $0,200^{*}$ & $0,200^{*}$ & $0,200^{*}$ \\
39 & 0,000 & 0,035 & 0,000 & $0,200^{*}$ & $0,200^{*}$ & $0,200^{*}$ \\
41 & 0,007 & 0,005 & 0,000 & $0,200^{*}$ & $0,200^{*}$ & $0,200^{*}$ \\
\hline
\end{tabular}

DAC - dias após corte; $\mathrm{p}>0,05$, não se rejeita $\mathrm{H}_{0}$ : normalidade dos dados.

*Limite inferior para a verdadeira significância de normalidade

Verificou-se que, em todas as camadas não ocorreu diferença significativa da $\theta$ entre as datas avaliadas ( $p>0,05)$, todavia, tendeu aumentar com a profundidade $(0,07 ; 0,072$; 0,078; 0,086; 0,090; e, $0,095 \mathrm{~m}^{3} \mathrm{~m}^{-3}$, respectivamente, $0,00-0,10 ; 0,10-0,20 ; 0,20-$ 0,30; 0,40-0,50; e, 0,50-0,60 m) (Figura 2A). Resultados similares foram citados por Araújo Primo et al. (2015) em um cultivo de palma forrageira em sequeiro, quando a $\theta$ aumentou com a profundidade, porém não se diferenciou entre as camadas a cada $0,10 \mathrm{~m}$ até $0,70 \mathrm{~m}$.

Por sua vez, os maiores coeficientes de variação (CV) foram verificados nas camadas mais profundas $(0,30-0,40 \mathrm{~m}, 0,40-0,50 \mathrm{~m}$ e
0,50-0,60 m, Figura 2B), onde os conteúdos de argila $\left(75,80 ; 95,10\right.$ e $\left.120,40 \mathrm{~g} \mathrm{~kg}^{-1}\right)$, silte (279,10, 297,90 e 304,60 $\left.\mathrm{g} \mathrm{kg}^{-1}\right)$ e porosidades totais foram mais elevados $(39,48 ; 42,58$ e 46,00\%) (Tabela 1). Nessas camadas, o sistema radicular da palma possui maior densidade (ARAÚJO PRIMO et al., 2015).

De modo geral, ao longo do tempo, a CV da $\theta$ foi classificada como média (10-20\%), de acordo com a classificação de Warrick e Nielsen (1980), mesmo quando houve irrigação $(6 \mathrm{~mm})$ após o dia 19 de junho de 2012 , com a $\theta$ da camada mais superficial $(0,0-$ 0,10 m), apresentando CV próximo de $30 \%$ (Figura 2B). 
Dias após corte (DAC)

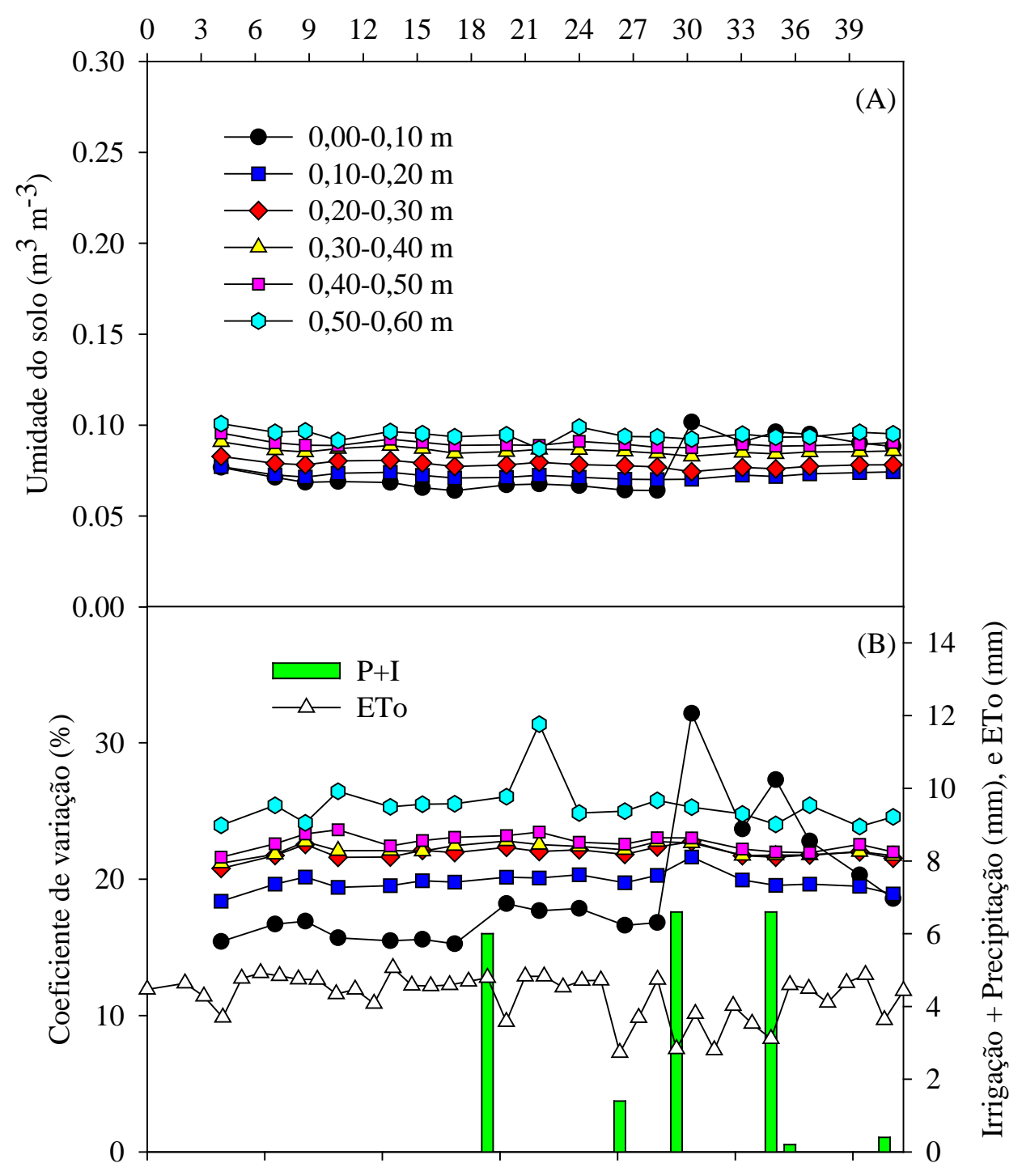

28-5-12 $\quad 04-6-12 \quad 11-6-12 \quad 18-6-12 \quad 25-6-12 \quad 02-7-12 \quad 09-7-12$

Data

Figura 2. (A) Variação da umidade em seis camadas (0,00-0,10; 0,10-0,20; 0,20-0,30; 0,40-0,50; e, 0,50-0,60 m) de um argissolo vermelho amarelo cultivado com palma forrageira; (B) Coeficiente de variação da umidade do solo e valores diários da evapotranspiração de referência (ETo) e precipitação mais irrigação $(\mathrm{P}+\mathrm{I})$

Os valores da diferença relativa na posição i $\left(\delta_{\mathrm{ij}}(\theta)\right)$ para as seis camadas do solo (0,00- 0,10 a $0,50-0,60 \mathrm{~m})$ revelaram que os pontos que apresentaram menor dispersão espacial por camada foram: o ponto 18 na camada de 0,00-0,10 m (Figura 3A); o ponto 19 na camada de 0,10-0,20 m (Figura 3B); o ponto 52 na camada de 0,20-0,30 m (Figura
3C); o ponto 57 nas camadas de 0,30-0,40 m (Figura 3D) e 0,40-0,50 m (Figura $3 \mathrm{E}$ ); e o ponto 6 na camada de 0,50 0,60 m (Figura 3F). Moreira et al. (2014) citam que, no monitoramento futuro da $\theta$, os pontos com menor diferença relativa são os que deverão ser mais adotados. 


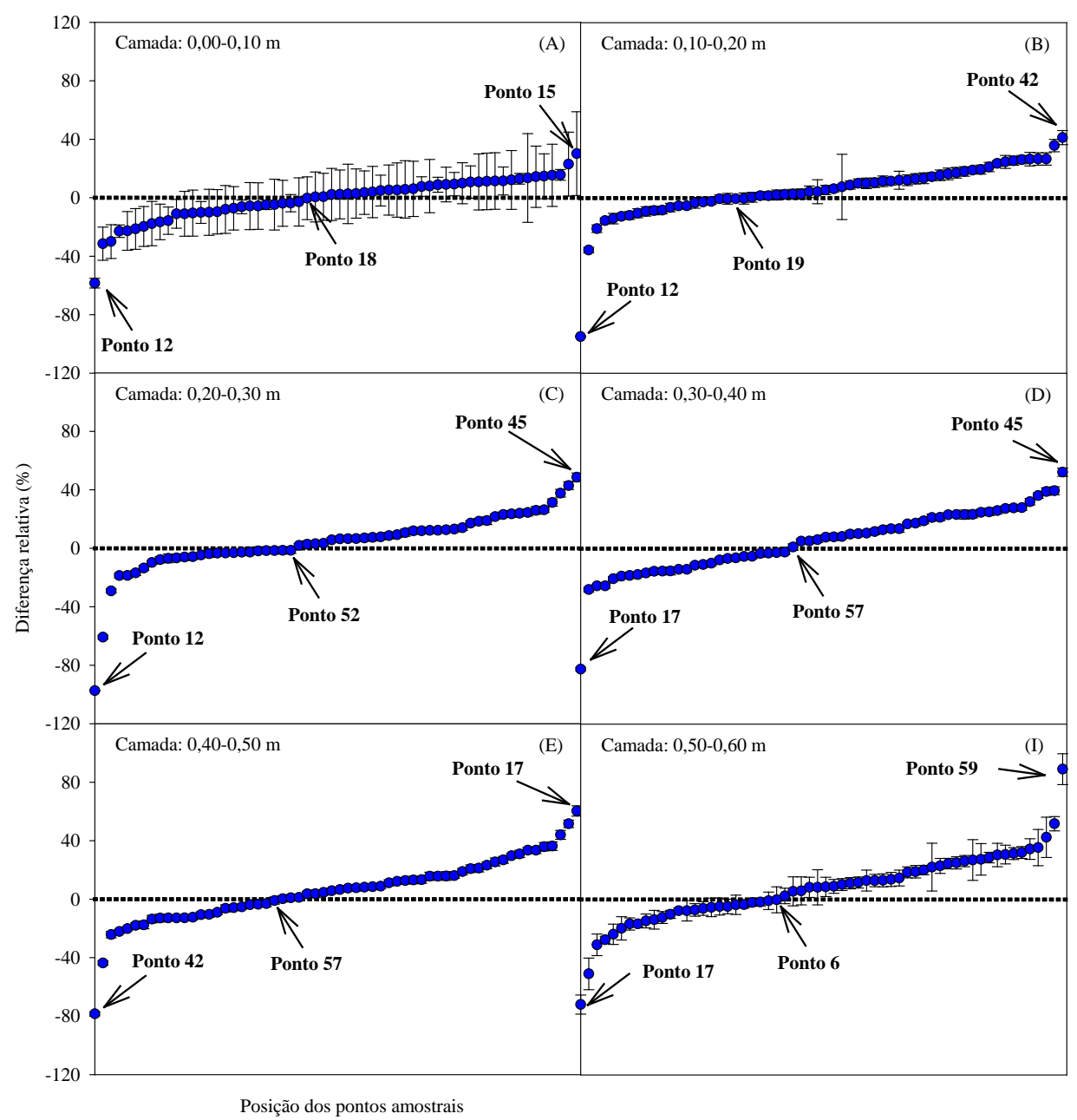

Figura 3. Diferença relativa da posição e o desvio padrão da umidade do solo $(\theta)$ de seis camadas do perfil $(0,00-0,10 ; 0,10-0,20 ; 0,20-0,30 ; 0,40-0,50 ;$ e, $0,50-0,60 \mathrm{~m})$ de um argissolo vermelho amarelo cultivado com palma forrageira

Percebe-se que, apenas duas camadas do solo $(0,30-0,40 \mathrm{~m}$, Figura 3D; e 0,40-0,50 m, Figura 3E) apresentaram a mesma posição de baixa dispersão da $\theta$ (ponto 57), indicando pouca convergência espacial desta variável nas demais profundidades. Melo Filho e Libardi (2005) citam que o bom planejamento agrícola depende da alta convergência da $\theta$ entre as posições e camadas da área, uma vez que garante a estabilidade espaço-temporal da $\theta$.

Na Figura 3A, observa-se que os pontos 12 e 15 , na camada $0,00-0,10 \mathrm{~m}$, foram aquelas posições que mais subestimaram e superestimaram a $\theta$, respectivamente, com valores de $\delta_{\mathrm{ij}}(\theta)$ iguais a $58,41 \%$ e $30,29 \%$, nessa ordem. As maiores discrepâncias na camada 0,10-0,20 m ocorreram nos pontos 12 e 42 (Figura 3B), com valores de 94,74\% e
41,45\%, enquanto, os pontos 12 e 45 na camada $0,20-0,30 \mathrm{~m}$, foram os de maiores magnitudes (97,37\% e 48,70\%, Figura 3C).

Nas camadas mais profundas, os pontos 17 e 45 promoveram $\delta_{\mathrm{ij}}(\theta)$ de $82,42 \%$ e $52,42 \%$ na camada 0,30-0,40 m (Figura 3D). Por sua vez, na camada 0,40-0,50 m (Figura 3E), os pontos 17 e 42, com valores de 78,40\% e $60,49 \%$ foram os mais discrepantes, enquanto, na camada 0,50-0,60, foram verificadas nos pontos 17 e 59 (71,82 e 89,30\%, Figura 3F).

Logo, os pontos 12 e 17 e, 42 e 45 foram os que subestimaram e superestimaram, respectivamente, a $\theta$, logo, não devem ser recomendados na medição futura da $\theta$ dentro da área de estudo.

Os valores dos coeficientes de correlação de Spearman $\left(\mathrm{r}_{\mathrm{s}}\right)$ foram significativos entre os 
intervalos de medições em todas as profundidades, indicando dependência temporal da $\theta$. Todavia, percebe-se que na camada de 0,00-0,10 a partir do dia 29, os valores foram baixos, embora significativos (Tabela 3), como resultados dos eventos de irrigação e de chuva. Na Tabela 3 foram apresentados apenas os valores de rs da $\theta$ entre as datas de monitoramento de duas camadas (0,00-0,10 m e 0,50-0,60 m), por causa da magnitude e significância dos valores serem similares entre a maioria das camadas $(0,10-$ $0,20 \mathrm{~m}$ a $0,50-0,60 \mathrm{~m})$.

Tabela 3. Coeficientes de correlação de Spearman da umidade do solo entre as datas de monitoramento para as camadas 0,00-0,10 m e 0,50-0,60 m, em uma área de cultivo de palma forrageira no Semiárido brasileiro. Todas as correlações foram significativas pelo menos a $5 \%$

\begin{tabular}{|c|c|c|c|c|c|c|c|c|c|c|c|c|c|c|c|c|c|c|}
\hline Camada & DAC & 4 & 6 & 8 & 11 & 13 & 15 & 18 & 20 & 22 & 25 & 27 & 29 & 32 & 34 & 36 & 39 & 41 \\
\hline \multirow{17}{*}{$\begin{array}{c}0,00- \\
0,10\end{array}$} & 1 & 0,965 & 0,953 & 0,961 & 0,943 & 0,911 & 0,912 & 0,874 & 0,873 & 0,879 & $\begin{array}{l}0,888 \\
\end{array}$ & 0,871 & 0,324 & 0,356 & 0,377 & 0,389 & 0,405 & 0,419 \\
\hline & 4 & & 0,969 & 0,975 & 0,960 & 0,939 & 0,933 & 0,893 & 0,897 & 0,912 & 0,905 & 0,893 & 0,276 & 0,315 & 0,311 & 0,327 & 0,353 & 0,369 \\
\hline & 6 & & & 0,981 & 0,963 & 0,944 & 0,936 & 0,886 & 0,884 & 0,901 & 0,902 & 0,891 & 0,274 & 0,311 & 0,301 & 0,316 & 0,345 & 0,368 \\
\hline & 8 & & & & 0,986 & 0,972 & 0,968 & 0,914 & 0,921 & 0,931 & 0,932 & 0,924 & 0,311 & 0,349 & 0,318 & 0,339 & 0,371 & 0,386 \\
\hline & 11 & & & & & 0,988 & 0,984 & 0,915 & 0,922 & 0,942 & 0,948 & 0,944 & 0,317 & 0,350 & 0,310 & 0,337 & 0,365 & 0,379 \\
\hline & 13 & & & & & & 0,988 & 0,915 & 0,929 & 0,951 & 0,957 & 0,957 & 0,291 & 0,325 & 0,272 & 0,298 & 0,334 & 0,345 \\
\hline & 15 & & & & & & & 0,919 & 0,934 & 0,951 & 0,965 & 0,963 & 0,333 & 0,363 & 0,307 & 0,336 & 0,369 & 0,379 \\
\hline & 18 & & & & & & & & 0,991 & 0,966 & 0,955 & 0,955 & 0,376 & 0,419 & 0,358 & 0,389 & 0,430 & 0,443 \\
\hline & 20 & & & & & & & & & 0,980 & 0,974 & 0,970 & 0,358 & 0,402 & 0,333 & 0,362 & 0,405 & 0,419 \\
\hline & 22 & & & & & & & & & & 0,978 & 0,977 & 0,292 & 0,336 & 0,275 & 0,303 & 0,344 & 0,358 \\
\hline & 25 & & & & & & & & & & & 0,992 & 0,351 & 0,385 & 0,322 & 0,350 & 0,388 & 0,403 \\
\hline & 27 & & & & & & & & & & & & 0,357 & 0,391 & 0,331 & 0,359 & 0,397 & 0,408 \\
\hline & 29 & & & & & & & & & & & & & 0,982 & 0,944 & 0,955 & 0,960 & 0,955 \\
\hline & 32 & & & & & & & & & & & & & & 0,941 & 0,958 & 0,969 & 0,962 \\
\hline & 34 & & & & & & & & & & & & & & & 0,994 & 0,981 & 0,979 \\
\hline & 36 & & & & & & & & & & & & & & & & 0,990 & 0,986 \\
\hline & 39 & & & & & & & & & & & & & & & & & 0,989 \\
\hline \multirow[t]{18}{*}{ Camada } & DAC & 4 & 6 & 8 & 11 & 13 & 15 & 18 & 20 & 22 & 25 & 27 & 29 & 32 & 34 & 36 & 39 & 41 \\
\hline & 1 & 0,753 & 0,689 & 0,774 & 0,778 & 0,766 & 0,763 & 0,770 & 0,721 & 0,750 & 0,740 & 0,749 & 0,751 & 0,769 & 0,752 & 0,745 & 0,784 & 0,752 \\
\hline & 4 & & 0,788 & 0,942 & 0,963 & 0,982 & 0,979 & 0,980 & 0,876 & 0,909 & 0,970 & 0,983 & 0,961 & 0,980 & 0,968 & 0,988 & 0,983 & 0,966 \\
\hline & 6 & & & 0,787 & 0,824 & 0,812 & 0,808 & 0,823 & 0,790 & 0,843 & 0,821 & 0,808 & 0,796 & 0,821 & 0,803 & 0,800 & 0,804 & 0,780 \\
\hline & 8 & & & & 0,933 & 0,953 & 0,955 & 0,950 & 0,884 & 0,896 & 0,923 & 0,949 & 0,972 & 0,948 & 0,968 & 0,943 & 0,941 & 0,959 \\
\hline & 11 & & & & & 0,968 & 0,975 & 0,975 & 0,872 & 0,936 & 0,945 & 0,968 & 0,960 & 0,975 & 0,957 & 0,964 & 0,969 & 0,943 \\
\hline & 13 & & & & & & 0,984 & 0,985 & 0,884 & 0,931 & 0,961 & 0,987 & 0,965 & 0,986 & 0,972 & 0,976 & 0,976 & 0,958 \\
\hline & 15 & & & & & & & 0,994 & 0,901 & 0,916 & 0,970 & 0,992 & 0,981 & 0,991 & 0,973 & 0,985 & 0,985 & 0,965 \\
\hline & 18 & & & & & & & & 0,902 & 0,924 & 0,972 & 0,994 & 0,977 & 0,996 & 0,975 & 0,979 & 0,990 & 0,969 \\
\hline & 20 & & & & & & & & & 0,837 & 0,928 & 0,896 & 0,905 & 0,902 & 0,906 & 0,882 & 0,886 & 0,897 \\
\hline & 22 & & & & & & & & & & 0,886 & 0,915 & 0,899 & 0,928 & 0,913 & 0,910 & 0,921 & 0,897 \\
\hline & 25 & & & & & & & & & & & 0,974 & 0,952 & 0,971 & 0,949 & 0,972 & 0,975 & 0,954 \\
\hline & 27 & & & & & & & & & & & & 0,976 & 0,995 & 0,975 & 0,981 & 0,986 & 0,968 \\
\hline & 29 & & & & & & & & & & & & & 0,974 & 0,987 & 0,965 & 0,970 & 0,979 \\
\hline & 32 & & & & & & & & & & & & & & 0,976 & 0,984 & 0,989 & 0,966 \\
\hline & 34 & & & & & & & & & & & & & & & 0,963 & 0,967 & 0,982 \\
\hline & 36 & & & & & & & & & & & & & & & & 0,984 & 0,961 \\
\hline & 39 & & & & & & & & & & & & & & & & & 0,973 \\
\hline
\end{tabular}

DAC - Dias após corte

Na condição de sequeiro, os altos e significativos valores de $\mathrm{r}_{\mathrm{s}}$ indicam manutenção da $\theta$, ou seja, a persistência do solo em armazenar água, fornecendo padrões temporais mais estáveis sobretudo a partir da segunda camada. Naturalmente, eventos de irrigação e de chuva podem promover baixas correlações entre os valores de $\theta$, as quais medidas em dias subsequentes. Este mesmo comportamento foi destaque no trabalho desenvolvido por Zhang e Shao (2013). 
Os modelos e os parâmetros dos semivariogramas ajustados para a $\theta$ nas camadas $\quad 0,00-0,10 ; \quad 0,10-0,20 ; \quad 0,20-0,30$; 0,30-0,40; $0,40-0,50$ e $0,50-0,60 \mathrm{~m}$, respectivamente, foram: Gaussiano $(\mathrm{C} 0=$ $3,20 \cdot 10^{-5} ; \mathrm{C} 0+\mathrm{C}=2,22 \cdot 10^{-4}$; a $(\mathrm{m})=70,49$ e $\left.\mathrm{R}^{2}=0,971\right)$; Gaussiano $\left(\mathrm{C} 0=4,40.10^{-5} ; \mathrm{C} 0+\right.$ $\mathrm{C}=4,40.10^{-5}$; $\mathrm{a}(\mathrm{m})=127,83$ e $\left.\mathrm{R}^{2}=0,925\right)$; Exponencial $\left(\mathrm{CO}=1,00.10^{-6} ; \mathrm{C} 0+\mathrm{C}=2,10.10^{-}\right.$ 3; a $(\mathrm{m})=605,40$ e $\left.\mathrm{R}^{2}=0,932\right)$; Gaussiano (C0 $=5,30 \cdot 10^{-5} ; \mathrm{C} 0+\mathrm{C}=1,12 \cdot 10^{-3} ; \mathrm{a}(\mathrm{m})=105,14$ e $\left.\mathrm{R}^{2}=0,987\right)$; Exponencial ( $\mathrm{C} 0=1,00.10^{-6}$; $\mathrm{C} 0$ $+\mathrm{C}=2,89 \cdot 10^{-3}$; a $(\mathrm{m})=632,70$ e $\left.\mathrm{R}^{2}=0,953\right) \mathrm{e}$

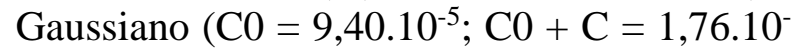
3; $\mathrm{a}(\mathrm{m})=119,68$ e $\left.\mathrm{R}^{2}=0,940\right)$.

Destaca-se que os alcances obtidos diferiram muito entre si, com valor mais baixo na camada de 0,00-0,10 m (70,49 m) e mais alto na camada de $0,40-0,50 \mathrm{~m}(632,70 \mathrm{~m})$. Por sua vez, as camadas de 0,20-0,30 m e 0,40-0,50 $\mathrm{m}$ tiveram maior relação espacial, uma vez que apresentaram alcances semelhantes de $605,40 \mathrm{~m}$ e $632,70 \mathrm{~m}$, respectivamente. Oliveira Filho et al. (2015) obtiveram alcances na faixa de 117 a 139 m. Esta dinâmica, segundo os autores, está relacionada à variação intrínseca das características do solo, como textura e mineralogia.

Os mapas da distribuição espacial da $\theta$ permitem visualizar as mudanças no comportamento horizontal da $\theta$ e, vertical, ao longo do perfil do solo (Figura 4).
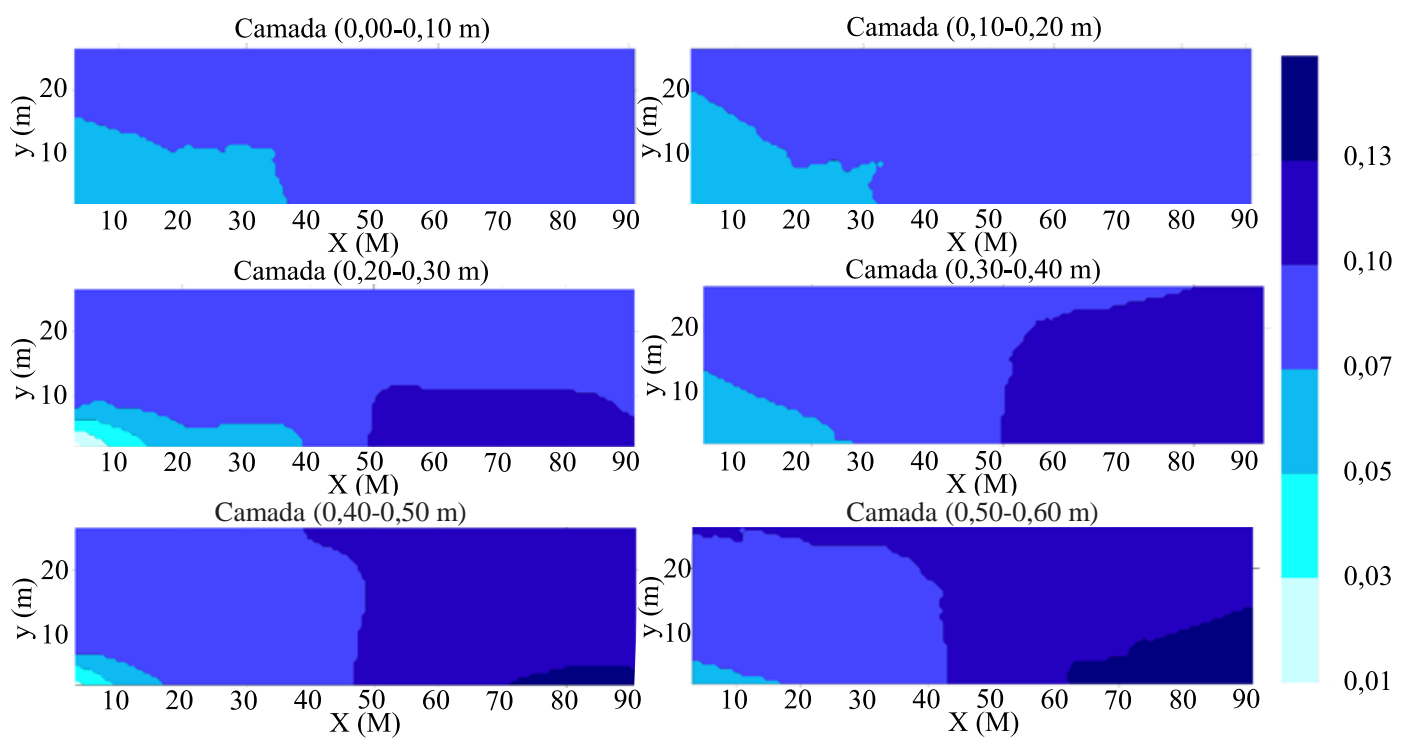

Figura 4. Mapas da umidade do solo de seis camadas $(0,00-0,10 ; 0,10-0,20 ; 0,20-0,30 ; 0,40-0,50 ;$ e, 0,50-0,60 $\mathrm{m}$ ) de um argissolo vermelho amarelo cultivado com palma forrageira.

Nota-se que os maiores valores de $\theta$ encontram-se a partir da camada de 0,30-0,40 $\mathrm{m}$, que atingem magnitudes próximas a 0,10 $\mathrm{m}^{3} \mathrm{~m}^{-3}$. As discrepâncias espaciais foram resultantes da diferença de cota dentro da área de estudo (máximo 0,50 m, ou seja, 0,6\% de declividade), os quais os pontos de menor cota tendem a possuir maiores $\theta$, em decorrência do favorecimento dos fluxos de água, oriundos das áreas mais elevadas. Esta afirmativa também foi feita por Ávila et al. (2010). No presente estudo, estes pontos estão bem associados com as posições que promoveram maiores estabilidades temporais (Figura 3).

\section{CONCLUSÕES}

A $\theta$ ao longo do perfil de um argissolo vermelho amarelo cultivado com palma forrageira demonstrou dispersão média $(12 \leq$ $\mathrm{CV} \leq 62 \%$ ), mesmo depois da realização de eventos de irrigação.

A técnica da diferença relativa da posição mostrou que a dispersão espacial da $\theta$ dependeu da camada do solo.

A matriz de correlação de Spearman detectou estabilidade temporal da $\theta$ em todas as camadas do perfil do solo, 
porém mais intensa nas maiores profundidades.

A geoestatística revelou dependência entre a $\theta$ em um cultivado com palma forrageira e a cota da posição de medição, e estreita relação aos pontos com maior estabilidade temporal.

\section{AGRADECIMENTOS}

À Coordenação de Aperfeiçoamento de Pessoal de Nível Superior (CAPES) e a Fundação de Amparo à Ciência e Tecnologia do Estado de Pernambuco (FACEPE).

\section{REFERÊNCIAS BIBLIOGRÁFICAS}

ALLEN, R. G et al. Crop evapotranspiration: guidelines for computing crop water requirements. 1. ed. Rome: FAO, 1998. 300 p. (FAO. Irrigation and Drainage Paper, 56)

ARAÚJO PRIMO, J. T.; SILVA, T. G. F.; SILVA, S. M. S.; MOURA, M. S. B.; SOUZA, L. S. B. Calibração de sondas capacitivas, funções físico-hídricas e variação do armazenamento de água em um argissolo cultivado com palma forrageira. Revista Ceres, v. 62, n. 1, p. 20-29, 2015.

ÁVILA, L. F.; MELLO, C. R.; MELLO, J. M.; SILVA, A. M. Padrão espaço-temporal da umidade volumétrica do solo em uma bacia hidrográfica com predominância de latossolos. Revista Brasileira de Ciência do Solo, v. 35, n. 5, p. 1801-1810, 2011.

ÁVILA, L. F.; MELLO, C. R.; SILVA, A. M. Estabilidade temporal do conteúdo de água em três condições de uso do solo, em uma bacia hidrográfica da região da serra da Mantiqueira. MG. Revista Brasileira de Ciência do Solo, v. 34, n. 6, p. 2001-2009, 2010.

CASTRO, F. S.; PEZZOPANE, J. E. M.; CECILIO, R. A.; PEZZOPANE, J. R. M.; XAVIER, A. C. Avaliação do desempenho dos diferentes métodos de interpoladores para parâmetros do balanço hídrico climatológico. Revista Brasileira de Engenharia Agrícola e Ambiental, v. 14, n. 8, p. 871-880, 2010.

CHEN, T.; DE JEU, R.; LIU, Y.; VAN DER WERF, G.; DOLMAN, A. Using satellite based soil moisture to quantify the water driven variability in NDVI: A case study over main land Australia. Remote Sensing of Environment, v. 140, n. 1, p.330-338, 2014.

EMPRESA BRASILEIRA DE PESQUISA AGROPECUÁRIA. Sistema brasileiro de classificação de solos. 3. ed. Brasília: EMBRAPA, 2013. 353 p.

HE, B.; WANG, H.; HUANG, L.; LIU, J.; CHEN, Z. A new indicator of ecosystem water use efficiency based on surface soil moisture retrieved from remote sensing. Ecological Indicators, v. 75, n. 1, p. 10-16, 2017.

HERNÁNDEZ, A. F.; CASTILLO, I. O.; AMADOR, B. M.; HERNÁNDEZ, J. L. G.; TROYO-DIEGUEZ, E. Yield and physiological traits of prickly pear cactus 'nopal' (Opuntia spp.) cultivars under drip irrigation. Agricultural Water Management, v. 70, n. 2, p. 97-107, 2004.

HU, W.; SHAO, M.; HAN, F.; REICHARDT, K.; TAN, J. Watershed scale temporal stability of soil water content. Geoderma, v. 158, n. 34, p. 181-198, 2010.

JIA, X.; SHAO, M.; ZHU, Y.; LUO, Y. Soil moisture decline due to afforestation across the Loess Plateau, China. Journal of Hydrology, v. 546, n. 1, p. 113-122, 2017.

LEMOS FILHO, L. C. A. Estabilidade temporal e análise espacial do armazenamento de água no solo arenoso da região de Petrolina - PE. 2010. 163 f. Tese (Doutorado em Engenharia Agrícola) Universidade Federal de Lavras, MG.

MELO FILHO, J. F.; LIBARDI, P. L. Estabilidade temporal de medidas do teor e do potencial mátrico da água no solo em uma 
transeção. Revista Brasileira de Ciência do Solo, Viçosa, v. 29, n. 4, p. 497-506, 2005.

MOREIRA, N. B.; LIBARDI, P. L.; SALVADOR, M. M. S.; SOUSA, H. H. F. Análise espaço-temporal de componentes do balanço hídrico em um Latossolo. Revista Brasileira de Ciência do Solo, v. 38, n. 1, p. 60-72, 2014.

OLIVEIRA FILHO, F. X.; MIRANDA, N. D. O.; MEDEIROS, J. F.; SILVA, P. C.; MESQUITA, F. O.; COSTA, T. K. Zona de manejo para preparo do solo na cultura da cana-de-açúcar. Revista Brasileira de Engenharia Agrícola e Ambiental, v. 19, n. 2, p. 186-193, 2015.

PENG, F.; MU, M.; SUN, G. Responses of soil moisture to climate change based on projections by the end of the 21st century under the high emission scenario in the 'Huang-Huai-Hai Plain' region of China. Journal of Hydro-environment Research, v. 14, n. 1, p. 105-118, 2017.

QUEIROZ, M. G.; SILVA, T. G. F.; ZOLNIER, S.; SILVA, S. M. S.; SOUZA, C. A. A.; CARVALHO, H. F. S. Relações hídrico-econômicas da palma forrageira cultivada em ambiente Semiárido. Irriga, p. 141-154, n. Edição Especial, 2016.

RÖTZER, K.; MONTZKA, C.; VEREECKEN, H. Spatio-temporal variability of global soil moisture products. Journal of Hydrology, v. 522, n. 1, p. 187-202, 2015.

SENTEK. Diviner 2000 user guide. v. 1.21. Stepney: Sentek Pty Ltd, 2000. 88p
SILVA, T. G. F.; ARAÚJO PRIMO, J. T.; MORAIS, L. E. F.; DINIZ, W. J. S.; SOUZA, C. A. A. Crescimento e produtividade de clones de palma forrageira no semiárido e relações com variáveis meteorológicas. Revista Caatinga, v. 28, n. 2, p. 10-18, 2015 a.

SILVA, T. G. F.; ARAÚJO PRIMO, J. T.; MOURA, M. S. B.; SILVA, S. M. S.; MORAIS, J. E. F.; PEREIRA, P. C.; SOUZA, C. A. A. Soil water dynamics and evapotranspiration of forage cactus clones under rainfed conditions. Pesquisa Agropecuária Brasileira, v. 50, n. 7, p.515525, 2015b.

SOUZA, E. R.; MONTENEGRO, A. A. A.; MONTENEGRO, S. M. G.; MATOS, J. D. A. Temporal stability of soil moisture in irrigated carrot crops in Northeast Brazil. Agricultural Water Management, v. 99, n. 1, p. 26-32, 2011.

VACHAUD, G.; SILANS, A. P.; BALABANIS, P.; VAUCLIN, M. Temporal stability of spatially measured soil water probability density function. Soil Science Society of America Journal, v. 49, n. 1, p. 822-827, 1985.

ZHANG, P.; SHAO, M. Temporal stability of surface soil moisture in a desert area of northwestern China. Journal of Hydrology, v. 505, n. 1, p. 91-101, 2013.

ZUCCO, G.; BROCCA, L.; MORAMARCO, T.; MORBIDELLI, R. Influence of land use on soil moisture spatial-temporal variability and monitoring. Journal of Hydrology, v. 516, n. 1, p. 193-199, 2014. 